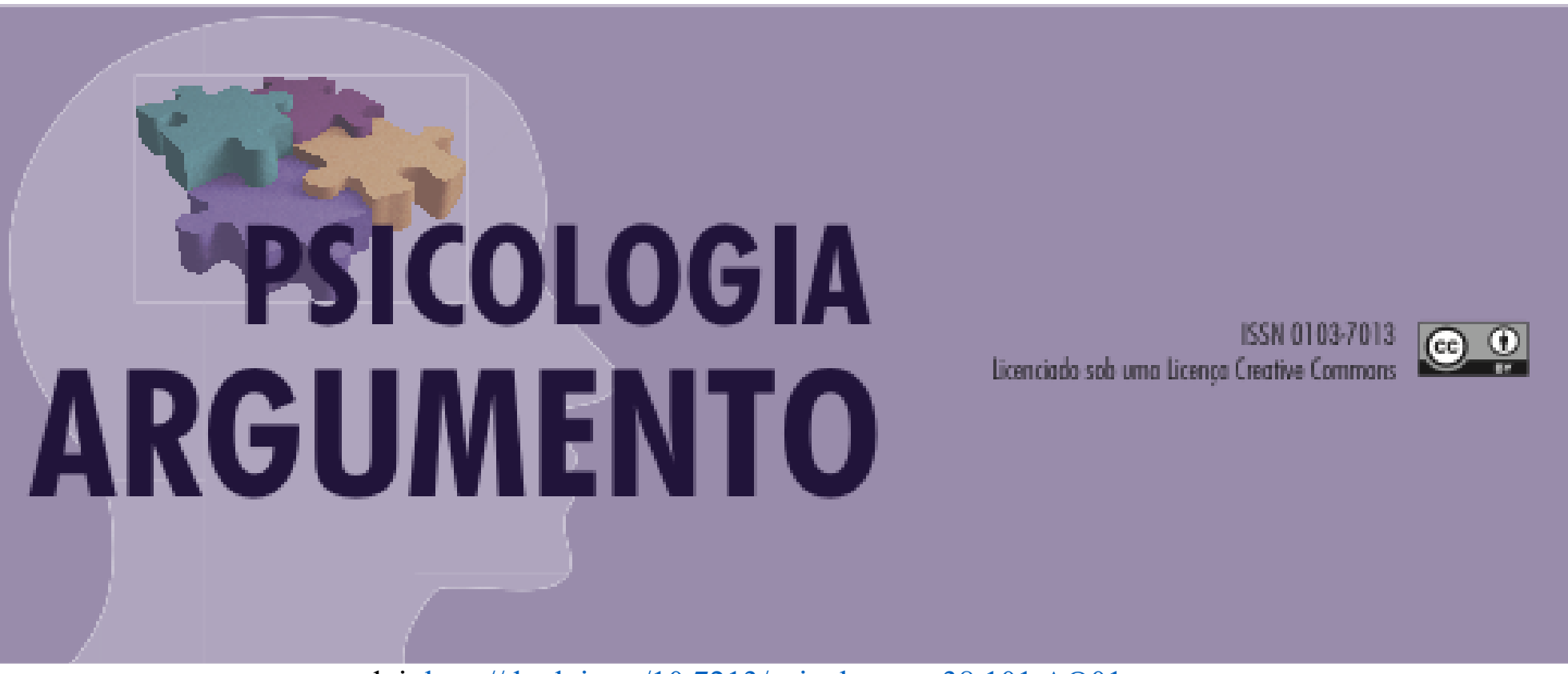

doi: http://dx.doi.org/10.7213/psicolargum.38.101.AO01

\title{
O comportamento verbal e não verbal na detecção da mentira: uma revisão bibliográfica
}

Verbal and nonverbal behavior in lie detection: a bibliographic review

El comportamiento verbal y no verbal en la detección de mentiras: una revisión bibliográfica

Pedro Eduardo Almeida Costa

Universidade Estadual de Londrina (UEL), e-mail: pedro.comportamental@gmail.com, orcid: https://orcid.org/0000-0001-5219-5338

Alex Eduardo Gallo

Universidade Estadual de Londrina (UEL), e-mail: aedgallo@uel.br, orcid:

https://orcid.org/0000-0002-3890-4270

\begin{abstract}
O presente estudo está inserido nas linhas de pesquisa que investigam a identificação do comportamento de mentir por meio da observação do comportamento verbal e não verbal. O objetivo principal foi a caracterização das pesquisas sobre a detecção de mentira por intermédio da observação, publicadas em âmbito nacional e internacional. A detecção da mentira por meio da observação das alterações do comportamento não verbal vem ganhando destaque atualmente e a bibliografia aponta que o número de informações obtidas com os métodos é maior, principalmente, quando se trata de interações em que podem ocorrer dissimulação de ideias, sentimentos e emoções. No sentido de elucidar questões pertinentes correspondentes às bibliografias no campo de pesquisa, o presente trabalho identificou, por meio de levantamento bibliográfico sistemático, estudos que abordaram a identificação da mentira por intermédio da observação do comportamento
\end{abstract}


não verbal. As buscas bibliográficas sobre o tema foram realizadas nas seguintes bases de dados: Scielo, Web of Science e PsicARTICLE (APA). Devido à especificidade da temática, julgou-se necessário a expansão da busca para os periódicos Journal of the Experimental Analysis of Behavior(JEAB) e Journal of Applied Behavior Analysis (JABA), bem como referências encontradas nos trabalhos obtidos e buscas na ferramenta de pesquisa Google Acadêmico. Foram incluídos na análise 46 artigos científicos empíricos. Observou-se um aumento no número de publicações entre os anos de 1999 e 2010, e, de acordo com a análise, resultados são variados e inconclusivos e a observação de comportamentos verbais e não verbais não podem servir como indicativos do comportamento de mentir.

Palavras-chave: Análise do Comportamento, Detecção de mentira, Comportamento verbal e não verbal.

\begin{abstract}
This study is part of a research field that investigate the identification of lying behavior by verbal and nonverbal behavior observation. The study aimed at characterizing researches about lying detection by observation, nationally and internationally published. Lying detection by observation of modifications on nonverbal behavior is gaining attention and published studies point at the higher number of information accessed using this method, especially interactions when behaviors, feelings, and emotions can be disguised. In order to elucidate pertinent questions about researches, the present study identified, by systematic reviewing, studies that investigated the lying detection by observation of nonverbal behavior. Bibliographic search took place on Scielo, Web of Science and PsicARTICLE (APA). Due limitations of research field, we expanded the search on Journal of the Experimental Analysis of Behavior (JEAB) and Journal of Applied Behavior Analysis (JABA), as well studies cited on recovered publications and Google Scholar. We analyzed 46 empirical researches. We found many publications between yeard 1999 and 2010, and results are varied and inconclusive, and observation of verbal and nonverbal behavior can not be used as significant instrument for lying detection.
\end{abstract}

Key-words: Behavior Analysis, Lying Detection, Verbal and nonverbal behavior.

\title{
Resumen
}

El presente estudio se inserta en las líneas de investigación que investigan la identificación del comportamiento mentiroso a través de la observación del comportamiento verbal y no verbal. El objetivo principal era caracterizar la investigación sobre detección de mentiras a través de la observación, publicada a nivel nacional e internacional. La detección de mentiras a través de la observación de cambios en el comportamiento no verbal está ganando importancia actualmente y la bibliografía señala que la cantidad de información obtenida con los métodos es mayor, especialmente cuando se trata de interacciones en las que puede ocurrir el ocultamiento de ideas y sentimientos. y emociones Con el fin de dilucidar las preguntas pertinentes correspondientes a las bibliografías en el campo de investigación, el presente trabajo identificó, a través de una encuesta bibliográfica sistemática, estudios que abordaron la identificación de mentiras a través de la observación del comportamiento no verbal. Se realizaron búsquedas bibliográficas sobre el tema en las siguientes bases de datos: Scielo, Web of Science y PsicARTICLE (APA). Debido a la especificidad del tema, se consideró necesario ampliar la búsqueda de las publicaciones periódicas Journal of the Experimental Analysis of Behavior (JEAB) y Journal of Applied Behavior Analysis (JABA), así como referencias 
encontradas en los trabajos obtenidos y búsquedas en la herramienta de investigación. Académico Google. 46 artículos científicos empíricos fueron incluidos en el análisis. Hubo un aumento en el número de publicaciones entre los años 1999 y 2010, y, según el análisis, los resultados son variados y no concluyentes y la observación del comportamiento verbal y no verbal no puede servir como indicativo del comportamiento mentiroso.

Palabras clave: Análisis de comportamiento, detección de mentiras, comportamiento verbal y no verbal.

\section{Introdução}

"O corpo fala: Juiz anula depoimento depois de analisar a linguagem corporal de testemunha', (O Globo, on-line, 2016). Essa matéria foi postada no portal O Globo, no dia 24 de fevereiro de 2016. Segundo ela, um juiz do estado do Rio Grande do Sul invalidou o depoimento de uma testemunha baseado em uma técnica contemporânea de coleta de dados por meio da observação da linguagem corporal. Na matéria, é destacado que as técnicas de observação podem ser de grande auxílio e a percepção dos sinais de alerta (gestos corporais que levantam dúvidas) apresentados pelo suspeito podem servir como pistas, indicando que pode ter algo além do que está sendo dito (ou omitido). A ação do juiz gerou algumas críticas e trouxe à tona discussões acerca da detecção de mentira, nas quais foi frisado que não existe técnica, teste ou método totalmente confiável ao investigar o comportamento de um suspeito de estar mentindo ou não. Todavia, casos como esse, em que é utilizada a técnica de observação do comportamento verbal e não verbal, devem ser cuidadosamente acompanhados de constantes discussões teóricas e práticas, uma vez que podem vir a causar prejuízos, tanto para o participante quanto para o aplicador. A partir das considerações destacadas, investigações que reúnem trabalhos relevantes nessa temática devem ser conduzidas com o objetivo de vir a servir como norteadoras de futuras pesquisas e ações, em que o uso da identificação do comportamento de mentir por meio da observação do comportamento verbal e não verbal (non-verbal/ nonverbal ${ }^{1}$ ) é aplicada.

Diversos processos comportamentais ocorrem durante a interação social. Os comportamentos sociais são desenvolvidos por meio da interação com a comunidade

\footnotetext{
${ }^{1}$ Neste trabalho, o termo não verbal corresponde aos termos em inglês "non-verbal" e "nonverbal".
} 
verbal e são mantidos ou não por suas consequências. Neste estudo, o comportamento em destaque é o de mentir. A mentira é considerada um comportamento operante e sua emissão tem por principal objetivo ter acesso a algum tipo de recompensa ou evitar punições (Ekman, 2009; Hübner, DaRocha \& Zotto, 2010). A mentira é um aspecto importante do comportamento humano, sendo classificada como uma habilidade social que colabora, de modo conveniente, com a convivência em sociedade. Tão importante quanto o desenvolvimento de um repertório comportamental adequado de mentir é a sua detecção. Dentre as técnicas de detecção de mentiras, destaca-se, aqui, a observação da emissão de comportamentos verbais e não verbais que podem contradizer o que está sendo vocalizado. Assim, sua identificação é importante para todo indivíduo que precisa de cooperação para assegurar sua sobrevivência (Honório, 2012).

De modo geral, as técnicas se baseiam em identificar, por meio da observação, frequências em que determinados comportamentos ocorrem, no intuito de verificar possíveis sinais que possam indicar estados emocionais e/ou intenção de mentir (De Paulo \& Rosenhal, 1979; Quinta \& Coelho, 2009; Zuckerman, De Paulo \& Rosenthal, 1981). Por meio de um estudo de revisão bibliográfica, De Paulo, Lindsay, Malone, Muhlenbruck, Charlton, \& Cooper (2003), destacaram que, ao mentir, o suspeito frequentemente: tem dificuldade para desenvolver o assuntou ou a história; desvia significativamente o olhar; movimenta com menos frequência braços, mãos e cabeça; e, por mais inusitado que pareça, tende a balançar/afirmar o oposto do que está sendo dito (ao responder sim, realiza movimentos de "não" ao movê-la horizontalmente). Assim, estabelecendo o fato de que os estados emocionais e o comportamento de mentir podem ser identificados por meio da observação de comportamentos não verbais.

O pioneiro a estudar a correlação entre expressões faciais e como estariam relacionadas ao mentir foi Charles Darwin (1872/2000). Em sua obra, Darwin apresenta que alguns movimentos (que podem ser emitidos involuntariamente) repetidos das expressões faciais podem estar relacionados ao alívio de algumas sensações, tornando-se hábitos e passíveis de identificação, já que têm estimulação direta do sistema nervoso, o que demonstra que, por mais habilidoso ou treinado que o suspeito (de mentir) seja, alguns sinais são passíveis de observação. Estudos que investigam a eficácia da detecção da mentira por meio da observação do comportamento verbal e não verbal originaram-se de uma pesquisa pioneira, realizada por Ekman e Friesen (1969), na qual os pesquisadores buscaram analisar diferenças do comportamento individualmente e como as alterações 
físicas (corporais) eram apresentadas quando os participantes mentiam. Posteriormente, Ekman e Friesen (1974) realizaram um estudo em que o objetivo era analisar entrevistas (gravadas) com 21 participantes suspeitos de estar mentindo durante o registro. Os autores concluíram, em ambos os experimentos, que os julgamentos foram baseados em alterações nos comportamentos verbais e não verbais, ressaltando, portanto, a importância e a eficácia desse tipo de técnica.

Atualmente, o campo da detecção de mentira por intermédio da observação de comportamentos verbais e não verbais está repleto de descobertas, indicando, em seus resultados, que as taxas de detecção excedem o acaso (Feeley \& deTurck, 2016; Frosina Logue, Book, Huizinga, Amos, \& Stark, 2018; De Turck, Harszlak, Bodhorn, \& Texter, 2016; Pereira, Lange, Shahid \& Swerts, 2017). No estudo conduzido por Frosina et al. (2018), constatou-se que, ao mentir, houve aumentos acentuados no piscar de olhos, diminuição de gesticulação das mãos e dificuldade em manter contato visual (olho no olho). Pereira, et al. (2017), demonstraram, por meio de um jogo virtual aplicado a crianças, que, ao mentir, os participantes sorriem mais quando comparados com os que relatam a verdade e que estratégias lúdicas (agente virtual) podem ser usadas como ferramenta para "extrair" a verdade. Assim, com os avanços tecnológicos e a ampliação nas áreas de investigação desse fenômeno, constata-se cada vez mais a efetividade da observação de comportamentos como forma de detecção de mentira.

\section{Objetivos}

As detecções da mentira por meio da observação de comportamentos verbais e não verbais podem oferecer pistas por intermédio de gestos ou de expressões faciais. Assim, esse método vem ganhando espaço e destaque em diferentes contextos em que é aplicado. Com base nas questões apresentadas, é de grande importância pesquisas que têm por norteadores localizar, identificar e oferecer sínteses de investigações anteriores sobre determinado problema. Dentre as metodologias, destacam-se trabalhos de revisões de literatura. Neste estudo, o objetivo consistiu em identificar e analisar, por meio de levantamento bibliográfico sistemático, estudos que abordaram a detecção da mentira por meio da observação do comportamento verbal e não verbal. 


\section{Método}

O levantamento bibliográfico foi realizado nas seguintes bases: Scielo, Web of Science e PsicARTICLE (APA). Devido à especificidade da temática, foram incluídos na busca os periódicos Journal of the Experimental Analysis of Behavior (JEAB) e Journal of Applied Behavior Analysis (JABA), referências encontradas nos trabalhos obtidos e buscas na ferramenta de pesquisa Google Acadêmico. A seleção das bases ocorreu após sondagens realizadas pelos pesquisadores que antecedeu a pesquisa e revistas específicas da Análise do Comportamento. Em todas as bases aplicaram-se os termos de busca, utilizadas pelo pesquisador, nos títulos, nos resumos, nas palavras-chaves e no assunto. O procedimento ocorreu no primeiro semestre de 2018.

A seleção dos registros foi feita nos seguintes critérios para sua inclusão. Os critérios foram amplos, sendo eles: (a) trabalhos empíricos cujo tema central era a aplicação de técnicas de observação do comportamento verbal e não verbal de mentir; (b) trabalhos nos idiomas português, inglês e espanhol. A escolha dos idiomas foi referente as bases de dados selecionadas e familiaridade dos autores. Os critérios de exclusão dos registros foram os seguintes: (a) pesquisas referentes a outros métodos de detecção de mentira e diversos à observação do comportamento verbal e não verbal (Polígrafo, Eletroencefalograma (EEG) e Ressonância Magnética Funcional (RMF)) que poderiam influenciar qualitativamente no tratamento e análise dos dados; (b) trabalhos repetidos ou incompletos. Não foi estabelecido um período específico para a busca.

Os trabalhos sobre os quais se obtiveram informações fazem parte do conhecimento científico produzido por diversos campos de conhecimento. Foram selecionados artigos empíricos brasileiros e internacionais, que abordaram o tema da detecção de mentira por meio da observação do comportamento verbal e não verbal. Foi realizada a inclusão, no banco de dados, de um protocolo de registro de bibliografia empírica representado nos seguintes campos: Assunto; participante (s); objetivo (s); metodologia; e principais resultados. Esses campos foram adicionados com o objetivo de coletar informações pertinentes e a fim de apresentar uma conclusão, informando as evidências, positivas e negativas, acerca da detecção de mentira por meio da observação do comportamento verbal e não verbal, com o intuito de aumentar o conhecimento a respeito das limitações e das vantagens desse procedimento. 


\section{Resultados e discussão}

Nos bancos de dados: Scielo, Web of Science e PsicARTICLE (APA), nos periódicos Journal of the Experimental Analysis of Behavior (JEAB) e Journal of Applied Behavior Analysis (JABA), e nas referências encontradas nos trabalhos obtidos e nas buscas na ferramenta de pesquisa Google, a partir da combinação das 3 combinações dos termos de busca para as bases nacionais e 4 para as internacionais. A Figura 1 apresenta o fluxograma que mostra o número de estudos selecionados a partir das buscas, o processo de seleção e o tratamento dos dados obtidos.

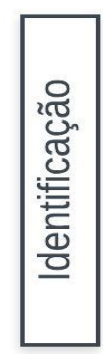

Referências identificadas:

Scielo $(\mathbf{n}=01)$; PsycARTICLES $(\mathbf{n}=16)$; Web of Science $(n=$

297); Journal of Applied Behavior Analysis (JABA) $(n=0)$; Journal of the Experimental Analysis of Behavior (JEAB) $(n=0)$
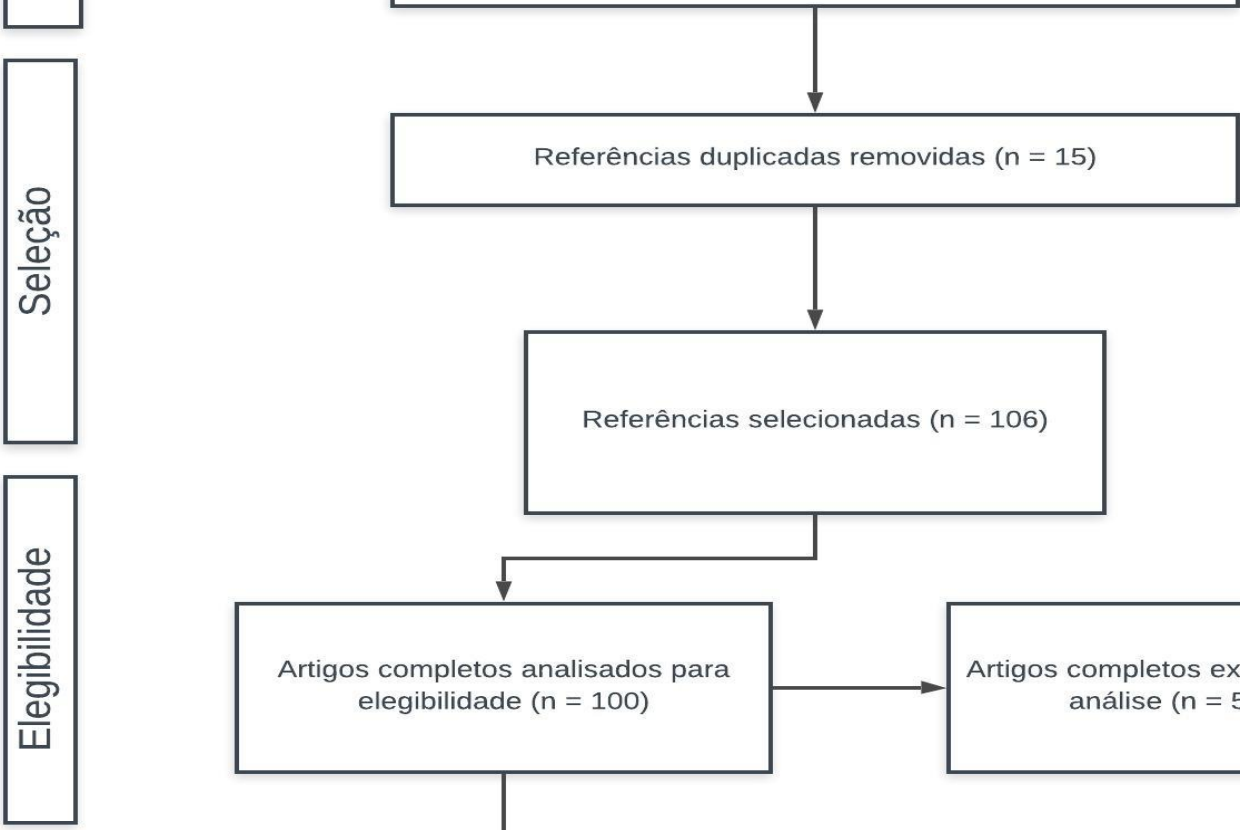

Referências selecionadas $(n=106)$
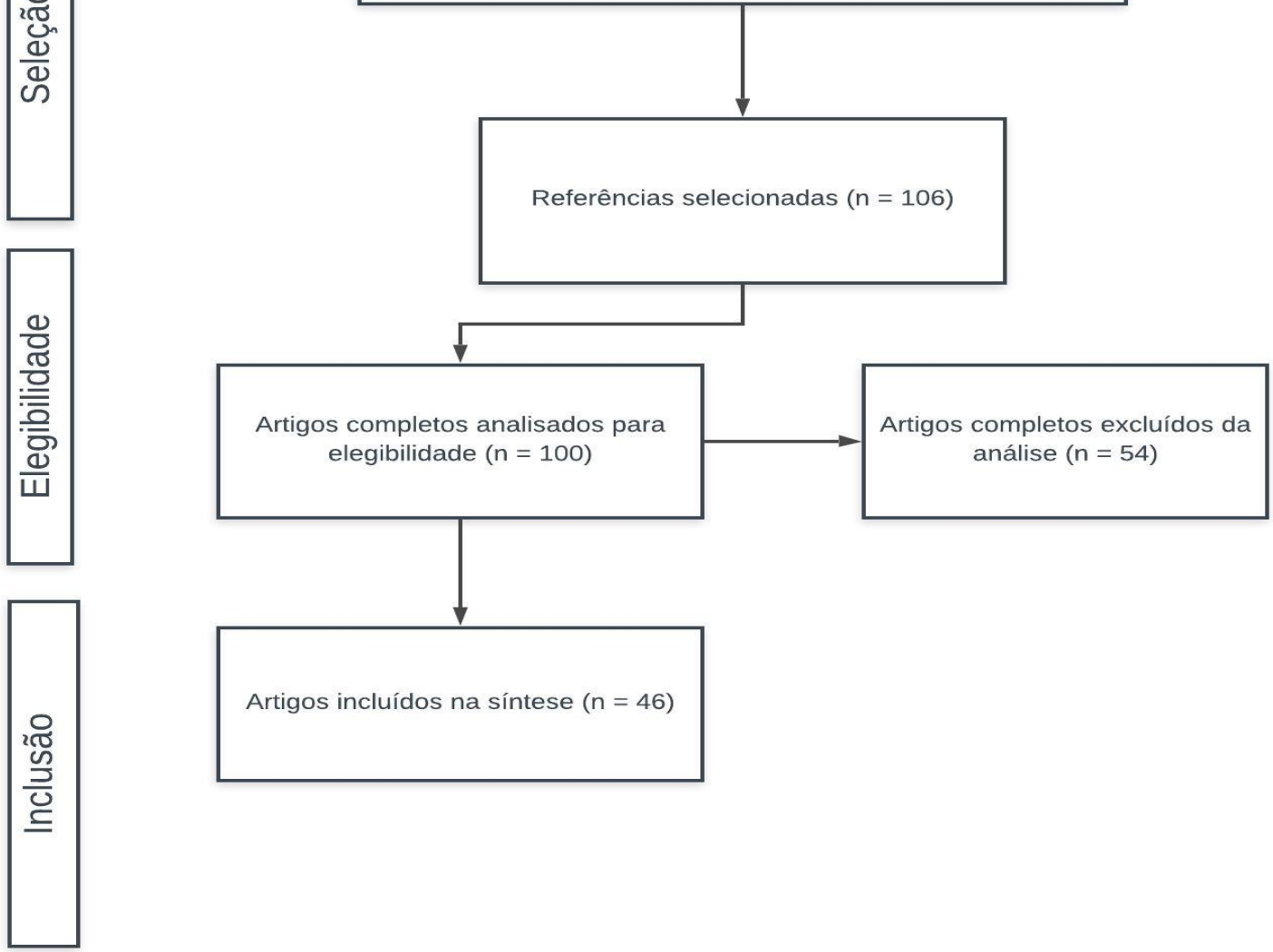

Figura 1. Processo de busca e tratamento dos trabalhos selecionados 
Depois de selecionados, os trabalhos foram registrados no banco de dados. A partir da demonstração das informações no instrumento, foram relacionados dados de identificação. As referências dos trabalhos obtidos e as buscas na ferramenta de pesquisa Google Acadêmico (representada como Outros) foram as que tiveram o maior número de trabalhos obtidos (32). Os artigos foram organizados quanto ao número de publicações por ano e observou-se um número maior de publicações a partir da década de 90 , sendo que o maior índice foi entre os anos 1999 e 2010. Os trabalhos também foram organizados quanto ao número de publicações por países, dentre eles, os com maior número de publicações foram os Estados Unidos (19), o Reino Unido (13) e o Canadá (7). Logo em seguida, Suécia, Portugal e Brasil (2), e Alemanha, Holanda, Paquistão, Singapura e Israel (1).

As revistas foram classificadas de acordo com o número de publicações incluído no trabalho. A revista com o maior número de publicação foi a Law and Human Behavior (8), seguida do Journal of Nonverbal Behavior (7). Após a constatação do número de publicações encontradas, resgatadas e adicionadas ao trabalho superior, quando comparado às outras revistas que tiveram seu(s) trabalho(s) incluso(s) na revisão, optouse por realizar uma busca com as combinações dos termos selecionados diretamente nas revistas (Law and Human Behavior e Journal of Nonverbal Behavior). Nenhum artigo, que já não havia sido incluído, encontrado respondeu aos critérios de inclusão.

Os autores foram identificados e classificados por número de publicações. Segundo esse levantamento, 87 autores diferentes foram identificados na presente pesquisa. Destes, Aldert Vrij (14) e Samantha A. Mann (8) foram os que tiveram o maior número de artigos empíricos incluídos no presente estudo. As instituições, por sua vez, foram classificadas de acordo com o número de publicações. Identificou-se um total de 61 instituições e a quantidade de publicações incluídas na análise. A partir da tabela, observa-se que a University of Portsmouth possui a maior parte de participação das publicações $(15(25 \%))$.

Quanto à caracterização dos participantes, notou-se que são as classes são variadas e que estudantes universitários tiveram maior participação (30 (46\%)) nos experimentos. Os Agentes de segurança também apresentaram grande número (16 (25\%)) de participação nos estudos. A efetividade da detecção da mentira por meio da observação do comportamento verbal e não verbal. Observou-se que a maior parte (29 (63\%)) dos resultados são expressivos (favoráveis à confirmação da efetividade de que a observação 
de determinados comportamentos (verbais e não verbais) pode servir como pistas do comportamento de mentir, facilitando, assim, sua detecção. Por último, dados referentes aos autores, participantes (número de participantes por estudo, idade média, sexo e tipo), informações relacionadas aos principais resultados e contou com a demonstração, isolada, da variável dependente (VD) e variável (eis) independente (s) (VI) de cada estudo foram identificadas.

Antes de iniciar a discussão, enfatiza-se que os trabalhos selecionados para o presente estudo são apenas aqueles que atenderam aos critérios adotados. Por consequência, não correspondem à totalidade de produções nacionais e internacionais sobre o tema da detecção da mentira. Foram considerados apenas artigos, sem restrições de ano, que realizaram experimentos empíricos com humanos e afirmaram testar a detecção da mentira por meio da observação do comportamento verbal e não verbal. Considerou-se importante essa observação para não ser necessário especificar a generalidade de cada análise ou uma conclusão mais adiante.

\section{Localização, identificação e seleção dos materiais nas bases de dados}

A seleção dos termos de busca e suas combinações foram essenciais, bem como permitiram a conclusão de que os termos "detecção de mentira" foram fundamentais para a localização dos artigos sobre o tema do presente estudo. A maioria dos artigos selecionados apresenta-os em suas palavras-chave e em seus resumos, colaborando, por muitas vezes, com a localização dos materiais sobre o tema e selecionados para o presente estudo.

Diversas considerações podem ser feitas a respeito de publicações sobre a detecção da mentira por meio da observação do comportamento verbal e não verbal. Em primeiro lugar, é importante notar que grande parte dos resultados encontrados foi em outras fontes (ferramenta de pesquisa do Google Acadêmico e inspeção das referências do material recuperado). A estratégia utilizada pelo pesquisador tinha por objetivo reunir o maior número de trabalhos possíveis que correspondessem aos critérios préestabelecidos de inclusão dos materiais. O número de materiais obtidos de outras referências pode estar relacionado ao maior raio de busca que a ferramenta de pesquisa Google Acadêmico possibilita, permitindo encontrar trabalhos que mesmo sem constar os termos de busca em seus títulos e em suas palavras-chave, abordavam o tema. 


\section{Número de publicações ao longo dos anos}

A análise da produção anual sobre o tema detecção da mentira por meio da observação do comportamento verbal e não verbal demonstra que o número de estudos não teve um aumento gradual ou estável ao longo dos anos (1969-2018). Observa-se um número superior de publicações entre os anos de 1999 e 2010, sendo que o ano de 2006 obteve o maior número, totalizando seis publicações. O fenômeno pode estar relacionado ao lançamento e/ou aumento da popularidade de livros sobre o tema e das séries de investigações policiais que ocorreram nesse período. Dentre as séries, pode-se destacar como exemplos: “CSI: Miami (2002)"; “CSI: New York (2004)”, “Criminal Minds (2005)", "Psych (2006)”, “The Mentalist (2008)", "Lie to Me (2009)”, dentre outras que abordam a temática entre os anos 1999 e 2010 (Adorocinema, 2019).

\section{O déficit das palavras-chave}

A tabulação das palavras-chave apresentada pelos autores dos artigos possibilitou a verificação de que a maior parte dos estudos não emprega diretamente o termo detecção da mentira (lie detection) no título (8) e nas palavras-chave (2), apesar de o estudo abordar diretamente o tema. Observou-se que palavras como deceit (3) e deception (15) são utilizadas como sinônimos de mentira. Hiposteniza-se que as palavras destacadas podem estar sendo utilizadas como meio de controlar possíveis variáveis estranhas, uma vez que a palavra mentira (lie) possui diferentes conotações (positivas e negativas) quando analisada em interação com a comunidade verbal. Dentre as palavras-chave mais frequentemente citadas, destacam-se a palavra deception e suas combinações, correspondendo à 33\% das palavras-chave classificadas. Constatou-se, ainda, que quase metade (25) dos estudos categorizados não continha palavras-chave associadas ao seu estudo. Supõe-se que pode ser devido a uma regra específica da revista na qual o trabalho foi publicado, ou pouca atenção tem sido dada nesse aspecto. Destaca-se que o uso das palavras-chave auxilia na localização e na identificação de um estudo, principalmente quando realizado em uma base de dados específica. Portanto, além da inclusão dessas palavras, sugere-se um grande cuidado na escolha desses termos, assim, buscar coerência com o tema e uma padronização com a literatura existente pode ser o mais adequado. 


\section{Número de publicações por países, instituições e a variedade dos estudos realizados}

Além do grande número de publicações sobre o tema, verificou-se que os estudos foram desenvolvidos em pelo menos 11 diferentes países. Ou seja, pesquisas que investigaram a detecção da mentira por meio da observação do comportamento verbal e não verbal são realizadas em quase todos os continentes do mundo. No entanto, a produção sobre o tema concentra-se nos seguintes países: Estados Unidos (19), Reino Unido (13), Canada (7), Suécia (2), Portugal (2) e Brasil (2). O maior número de publicações científicas foi observado nos Estados Unidos, possivelmente pela maior concentração de grandes universidades e de incentivos acadêmicos. Dentre as instituições estadunidenses, destaca-se a Florida International University (4), a University of California (2) e a University of San Francisco (2). Pode ser verificada também, com base no recorte das instituições destacadas, a variedade de pesquisas relacionadas ao tema, como examinar e avaliar estilos de entrevistas (Albrechtsen, Meissner \& Susa, 2009; Vrij, Kristen \& Fisher, 2007), testar hipóteses acerca do contato visual como facilitado na detecção da mentira (Vrij, Mann, Leal \& Fisher, 2010), avaliar o envolvimento e a efetividade e do julgamento de especialistas e amadores na detecção da mentira (Bond, 2009; De Paulo \& De Paulo, 1989; Ekman, O'Sullivan, Friesen \& Scherer, 1991; Feeley \& De Turck, 2016; De Turck, Harszlak, Bodhorn \& Texter, 2016; Forrest \& Feldman, 2000; Kassin \& Fong, 1999; Zuckerman \& Alton, 1984), testar técnicas específicas (Landry \& Brigham, 1992; Vrij, Mann \& Fisher, 2006), ampliar a compreensão do engano, examinando-o como um evento didático (Burgoon \& Buller, 1994), entre tantas outras.

A partir da tabulação e da classificação das revistas na base dedados, pode-se verificar que os números de periódicos são vastos e variados. Foram identificadas 24 revistas que publicaram sobre a detecção da mentira por intermédio da observação do comportamento verbal e não verbal. Dentre as revistas, destacou-se a Law and Human Behavior, que realizou oito publicações. Considerada uma revista multidisciplinar, ela tem por objetivo trazer discussões de questões recorrentes das relações entre o sistema legal, o processo legal, o comportamento humano e a lei. Na classificação, na segunda posição, encontra-se o Journal of Nonverbal Behavior, com sete publicações sobre o tema. Esse periódico, por sua vez, publica artigos empíricos originais referentes ao 
comportamento não verbal, abordando o comportamento não verbal e abrangendo os seguintes campos: proxêmica, paralinguagem, expressões visuais, interação face a face, contato visual e comportamento emocional não verbal, além de outros assuntos considerados relevantes para a compreensão científica dos comportamentos não verbais e dos processos envolvidos.

\section{Tipos de participantes por estudo: praticidade e utilidade}

Além das informações já apresentadas, os tipos de participantes foram identificados e, posteriormente, classificados individualmente, por participação em cada estudo. Nota-se predominância no envolvimento de estudantes universitários em pesquisas relacionadas à temática. Infere-se a possibilidade de (a) maior facilidade no engajamento de atividades, (b) flexibilização no horário para a coleta de dados, e (c) possível interesse em remuneração financeira e/ou outros benefícios (quando houver). Observou-se, também, que os agentes de segurança tiveram um número superior quando comparado aos outros tipos de participantes. Supõe-se que, por tratar-se de uma área em que existem interações com suspeitos, as habilidades de notar os sinais por meio da observação podem facilitar a detecção do engano (Castilho, 2011).

\section{Resultados positivos versus negativos: a detecção da mentira por meio da observação de comportamentos verbais e não verbais funciona?}

Foi registrada a efetividade da aplicação das técnicas de observação do comportamento verbal e não verbal como forma de detecção da mentira com base nos resultados descritos pelos autores. Ressalta-se que os resultados apresentados nesse estudo são baseados nos mesmos oferecidos pelos autores. Com base nos dados, 11\% (5) estudos não avaliaram diretamente a efetividade da observação do comportamento verbal e não verbal como forma de detecção da mentira. Já, 63\% (29) dos estudos exibiram, por meio dos resultados, confirmação para a efetividade de que a observação de determinados comportamentos (verbais e não verbais) podem colaborar com a detecção da mentira. Em contraste, $26 \%$ (12) trabalhos concluíram que essa forma de detectar o engano não se difere do acaso. Os resultados negativos para a efetividade corroboram com discussões realizadas acerca desse tipo de atividade, em que os comportamentos de observar 
determinados sinais podem não ser o suficiente ou não colaborar com a detecção do engano (Bond \& Uysal, 2007).

\section{O treino da detecção da mentira}

O treino é uma forma de aprendizagem e de aprimoramento de determinadas habilidades e pode ocorrer de diversas maneiras, como por exemplo: o controle de estímulo e treino discriminativo (Moreira, Oliveira, \& Hanna, 2017). Na detecção da mentira, enquanto um comportamento operante, também pode passar por processos que podem favorecer um desenvolvimento mais adequado/acurado tornando essa habilidade ou técnica mais efetiva. Estudos como os de Quinta \& Coelho (2009), Porter, et al. (2000), e Zuckerman et al. (1984) corroboram com o fato de que treinos com o feedback colaboram com o aumento do desempenho dos participantes durante o experimento. No estudo de Quinta e Coelho (2009), foi investigado e avaliado o efeito do treino com feedback disponibilizado após cada julgamento durante a atividade relacionada a detecção da mentira. Os resultados do estudo indicaram que o treino com feedback levou ao aumento do desempenho de todos os participantes (Quinta \& Coelho, 2009), corroborando com estudos em que foi investigado a mesma questão (Porter, Woodworth, \& Birt, 2000; Zuckerman et al., 1984). No entanto, foi também identificado um estudo em que os resultados indicaram que o treino pode ter afetado negativamente o desempenho dos participantes (Kassim \& Fong, 1999). Assim, sabe-se que o treino, na maioria dos estudos selecionados, favoreceu a aquisição e/ou aprimorar as habilidades de detecção da mentira.

\section{Protocolo Criteria-Based Content Analysis (CBCA) e o procedimento Statement Validity Analysis (SVA)}

O protocolo CBCA baseia-se na hipótese de que uma afirmação derivada da memória de uma experiência real, na qual existe um relato sincero da situação, se difere em conteúdo e qualidade de uma afirmação baseada em uma mentira, invenção ou fantasia (Vriji, Akehurst, Soukara, \& Bull, 2004; Vrij, Edward, Roberts, \& Bull, 2000; Landry, \& Brigham,1992). De acordo Griesel, Ternes, Schraml, Cooper e Yuille (2013), Vrij e Ganis (2014) e Machado, Silvano e Hutz (2015), para a avaliação de determinados 
comportamentos que podem servir como pistas para o relato enganoso ou verdadeiro, o CBCA se apoia em 19 critérios, classificados em diferentes agrupamentos, sendo eles: "Características gerais", "Conteúdos Específicos", "Conteúdos Referentes à Motivação" e "Elementos Específicos do Evento" (Machado et al., 2015).

Nos estudos identificados que fizeram o uso do CBCA, apresentam através dos resultados que a ferramenta pode facilitar a detecção da mentira. No estudo de Vrij, et al. (2006), foi destacado que a detecção do engano, utilizando o protocolo como instrumento, pode chegar a $60 \%$ de precisão. Os resultados de Vrij (2006) colabora com estudos anteriores conduzidos por Vrij, et al (2000) e Landry e Brigham (1992), no qual os participantes que tiveram acesso a esse protocolo, tiveram um desempenho superior aos demais, e estatisticamente maiores que o acerto ao acaso. No entanto, deve-se estar atento às limitações do instrumento, como por exemplo, a falta de um ponto de corte que precise, se a análise do conteúdo com base na versão, é verdadeiro ou falso (Machado et al., 2015). Assim, nota-se que o uso de protocolos, apesar de possuir limitações, podem auxiliar no julgamento da veracidade do testemunho por meio da observação de aspectos verbais e não verbais.

No Statement Validity Assessment/Analysis (SVA), pode ser compreendido como um método, que identifica aspectos relacionados a credibilidade de um relato. De acordo com Machado, et al. (2015), "A premissa principal do SVA é de que um testemunho derivado da memória de uma" experiência real difere em conteúdo e em qualidade de um testemunho baseado em fantasia ou invenção" (p. 36). O procedimento do SVA possui três partes: Entrevista Cognitiva, verificação de 19 critérios do CBCA e Lista de Controle da Validade do Testemunho (Griesel, et al., 2013; Machado, et al., 2015). Com base no estudo identificado, os autores Porter e Yuille (1996), concluíram por meio de seu experimento, que os resultados não tiveram muita expressividade, indicando que apenas 3 das $18(16,7 \%)$ pistas de engano testadas, diferenciaram as descrições verdadeiras e enganosas. Assim, com base nas considerações apresentadas, o uso de métodos e/ou protocolos específicos como uma ferramenta auxiliadora na detecção do engano, pode facilitar e aumentar a precisão na detecção da mentira, no entanto, deve-se estar atento, analisar e considerar diversos outros aspectos que podem estar interferindo nos dados coletados.

\section{Variáveis dependentes e independentes}


Cerca de 95,7\% (44) dos trabalhos buscaram compreender a detecção da mentira (VD) manipulando situações e contextos dos mais diversos (VI). As VIs, variaram da influência do uso contato visual (Vrij, et al., 2010), do piscar ocular (Leal \& Vrij, 2008), treinos (Quinta \& Coelho, 2009; Porter, Woodworth, \& Birt, 2000; Zuckerman, et al., 1984), análise e julgamentos de entrevistas (Vrij, et al., 2007; Vrij, Mann, Fisher, Leal, Milne, \& Bull, 2008; Rodrigues \& Arriaga, 2010), atividades diversas (Chan, Khader, Ang, Chin, \& Chai, 2016) até a manipulações mais complexas, como o estudo de Reinhard (2010), que controlou a tendência de processar informações (Need for cognition (NFC)). Um dos estudos (Albrechtsen, Meissner, \& Susa, 2009), não trouxe a detecção da mentira como variável dependente, porém, a manipulou para compreender os processamentos: intuitivo versus deliberativo. E um estudo teve apenas a variável independente localizada (Burgoon, \& Buller, 1994). Destaca-se que as variáveis foram demonstradas de acordo com o que os trabalhos apresentaram, foi evitado, pelos pesquisadores, inferências e julgamentos que poderiam interferir na tabulação.

\section{Considerações finais}

O objetivo do trabalho foi identificar, por meio de uma revisão de literatura sistemática, e analisar estudos que abordaram a detecção da mentira por meio da observação do comportamento verbal e não verbal. Enfatiza-se que o presente estudo teve por limitação os critérios de inclusão/exclusão. Por consequência, apenas os artigos que atenderam os critérios adotados foram incluídos, que por consequência, não correspondem à totalidade de produções relacionados a temática.

Diante do exposto e das variáveis levantadas no presente estudo, os dados indicados na literatura foram variados e não conclusivos. Ao observar sob perspectiva analítico-comportamental, não é possível operacionalizar os termos/expressões utilizados pelos autores apenas com a leitura de suas publicações. Além das informações apresentadas, notou-se que faltaram informações consideradas básicas nos estudos, como a definição da mentira ou do comportamento de mentir, a definição do comportamento verbal e não verbal (independentemente da área de pesquisa e campo de estudo), e até mesmo, em alguns casos, quais comportamentos identificados/propostos pelos autores são tratados como sinais de mentira. 
Apesar de o assunto não ser recente, e de existirem evidências convincentes acerca da efetividade das técnicas de detecção de mentiras por meio da observação do comportamento verbal e não verbal, com bases nos resultados deste estudo, ainda é prematuro chegar a qualquer tipo de conclusão. O que se sabe, é que pesquisas que envolvem protocolos específicos, como o CBCA e o SVA, apesar de possuir limitações, convergem em resultados expressivos de efetividade. Outro método que se mostra promissor é o treino da identificação de possíveis sinais que possam indicar o comportamento de mentira. Para futuros estudos de revisões bibliográficas relacionados a temática, sugere-se ampliar e refinar o uso de operadores booleanos e suas possíveis combinações para uma possível otimização dos resultados, o que poderia evitar números elevados de publicações com baixa ou nenhuma relevância para o trabalho.

Longe de pretender esgotar a discussão, deixam-se algumas questões que deveriam ser refletidas antes de se tomar qualquer conclusão e iniciar possíveis pesquisas sobre esse tipo de atividade. O que tem provocado tanta variedade nos resultados dos experimentos? Será que está claro para todos que estudam o comportamento de mentir, o comportamento verbal e o não verbal o que de fato eles significam?

\section{Referências}

Adorocinema. (2019). Todas as séries. Recuperado de http://www.adorocinema.com/series-tv/.

Albrechtsen, J. S., Meissner, C. A., \& Susa, K. J. (2009). Can intuition improve deception detection performance? Journal of Experimental Social Psychology, 45(4),1052-55. doi: 10.1016/j.jesp.2009.05.017.

Bond, G. D. (2009). Deception detection expertise. Law and Human Behavior, 32, 339351. doi: 10.1007/s10979-007-9110-z.

Burgoon, J. K., \& Buller, D. B. (1994). Interpersonal deception: III. Effects of deceit on perceived communication and nonverbal dynamics. Journal of Nonverbal Behavior, 18, 155-184. doi: 10.1007/BF02170076.

Chan, S., Khader, M., Ang, J., Chin, J., \& Chai, W. (2016). To behave like a liar: Nonverbal cues to deception in an asian sample. Journal of Police and Criminal Psychology, 31, 165-172. doi: 10.1007/s11896-015-9177-2. 
Darwin, C. (1872/2000). A expressão das emoções nos homens e nos animais. São Paulo, SP: Editora das Letras.

De Paulo, B. M., \& Rosenhal, R. (1979). Ambivalence, dscrepancy and deception in nonverbal communicatiion. In R. Rosenthal (Ed.), Skill in nonverbal communication (pp. 204-248). Cambridge, MA: Oelgeschlager, Gunn \& Hain.

De Paulo, B. M., Lindsay, J. J., Malone, B. E., Muhlenbruck, L., Charlton, K., \& Cooper, H. (2003). Cues to deception. Psychological Bulletin, 129, 74-118.

De Paulo, P. J., \& De Paulo, B. M. (1989). Can deception by salespersons and customers be detected through nonverbal behavioral cues? Journal of Applied Social Psychology, 19, 1552-1577. doi: 10.1111/j.1559-1816.1989.tb01463.x .

De Turck, M. A., Harszlak, J. J., Bodhorn, D. J., \& Texter, L.A. (2016). The effects of training social perceivers to detect deception from behavioral cues. Communication Quarterly, 38, 189-199. doi: 10.1080/01463379009369753.

Ekman, P. (2009). Telling lies: clues to deceit in the marketplace politics and marriage (3a ed.). New York, EUA: W.W. Norton.

Ekman, P., \& Friesen, W. V. (1969). Nonverbal leakage and clues to deception. Psychiatry, 32, 88-105. Recuperado de https://1ammce38pkj41n8xkp1iocwewpengine.netdna-ssl.com/wp-content/uploads/2013/07/Nonverbal-Leakage-AndClues-To-Deception.pdf.

Ekman, P., \& Friesen, W. V. (1974). Detecting deception from the body or face. Journal of Personality and Social Psychology, 29, 228-298. Recuperado de https://1 ammce38pkj41n8xkp1iocwe-wpengine.netdna-ssl.com/wpcontent/uploads/2013/07/Detecting-Deception-From-The-Body-Or-Face.pdf.

Ekman, P., \& O’Sullivan, M. (1991). Who can catch a liar? American Psychologist, 46, 913-920.

Feeley, T. H., \& Turck, M. A. de. (2016). Global cue usage in behavioral lie detection. Communication Quarterly, 43(4), 420-430. doi: 10.1080/01463379509369989.

Forrest, J. A., \& Feldman, R. S. (2000). Detecting deception and judges involvement: Lower task involvement leads to better lie detection. Personality and Social Psychology Bulletin, 26, 118-125. doi: 10.1177/0146167200261011. 
Frosina, P., Logue, M., Book, A., Huizinga, T., Amos, S. \& Stark, S. (2018). The effect of cognitive load on nonverbal behavior in the cognitive interview for suspects. Personality and Individual Differences, 130, 51-58. doi: 10.1016/j.paid.2018.03.012.

Griesel, D., Ternes, M., Schraml, D., Cooper, B. S., \& Yuille, J. C. (2013). The ABC's of CBCA: Verbal Credibility Assessment in Practice. In B. S. Cooper, D. Griesel \& M. Ternes (Eds.), Applied issues in investigating interviewing, eyewitness memory, and credibility assessment. New York, NY: Springer.

Honório, F. F. (2012). Precisão na detecção de mentiras: Investigação sobre o efeito da detecção indireta (Dissertação de mestrado em Ciências do Comportamento). Universidade de Brasília, Brasília, DF, Brasil.

Hübner, M. M., Da Rocha, G. M., \& Zotto, L. L. S. (2010). Mentira. In P. I. C. Gomide (Org.), Comportamento moral: Uma proposta para o desenvolvimento das virtudes (pp. 99-114). Curitiba, PR: Juruá.

Kassin, S. M., \& Fong, C. T. (1999). “I'm innocent!” Effects of training on judgments of truth and deception in the interrogation room. Law and Human Behavior, 23, 499-516. doi: 10.1023/A:1022330011811.

Landry, K., \& Brigham, J. C. (1992). The effect of training in criteria-based content analysis on the ability to detect deception in adults. Law and Human Behavior, 16, 663675. doi: 10.1007/BF01884022.

Leal, S., \& Vrij, A. (2008). Blinking during and after lying. Journal of Nonverbal Behavior, 32, 187-194. doi: 10.1007/s10919-008-0051-0.

Machado, P. V., Silvano, M. B., \& Hutz, C. S. (2015). Estudo exploratório sobre critérios de veracidade em relatos de eventos de vida: considerações para a perícia psicológica criminal de adultos. Aletheia, (47-48), 35-50. Recuperado de http://pepsic.bvsalud.org/scielo.php?script=sci_arttext\&pid=S141303942015000200004\&lng=pt\&tlng=pt.

Moreira, M. B., Oliveira, A., \& Hanna, E. S. (2017). Arranjo de estímulos em treino discriminativo simples com compostos e emergência de classes de estímulos equivalentes. Temas em Psicologia, 25(1), 351-367. Recuperado de https://dx.doi.org/10.9788/TP2017.1-19Pt. 
O Globo. (2016). O corpo fala: Juiz anula depoimento depois de analisar linguagem corporal de testemunha [on-line]. Recuperado de http://blogs.oglobo.globo.com/blogdo-moreno/post/o-corpo-fala-juiz-anula-depoimento-depois-de-analisar-linguagemcorporal-de-testemunha.html.

Pereira, M. S., Lange, J. de., Shahid, S., \& Swerts, M. (2017). A perceptual and behavioral analysis of facial cues to deception in interactions between children and a virtual agent. International Journal of Child-Computer Interaction, 15, 1-12. doi: 10.1016/j.ijcci.2017.10.003.

Porter, S., Woodworth, M., \& Birt, A. R. (2000). Truth, lies, and videotape: An investigation of the ability of federal parole officers to detect deception. Law and Human Behavior, 24, 643-658. doi: 10.1023/A:1005500219657.

Porter, S., \& Yuille, J. C. (1996). The language of deceit: An investigation of the verbal clues to deception in the interrogation context. Law and Human Behavior, 20, 443-459. doi: 10.1007/BF01498980.

Porter, S., Woodworth, M., \& Birt, A. R. (2000). Truth, lies, and videotape: An investigation of the ability of federal parole officers to detect deception. Law and Human Behavior, 24, 643-658. doi: 10.1023/A:1005500219657.

Quinta, N. C. C. de., \& Coelho, C. (2009). Contando e detectando mentiras: o efeito do feedback sobre o desempenho. Psicologia: Teoria e Pesquisa, 2(1), 137-145.

Reinhard, M. A. (2010). Need for cognition and the process of lie detection. Journal of Experimental Social Psychology, 46, 961-97 doi: 10.1016/j.jesp.2010.06.002.

Rodrigues, A., \& Arriaga, P. (2010). Haverá diferenças individuais na capacidade para detectar a mentira e a honestidade nos outros? Psicologia, 24(2), 43-60. Recuperado de http://www.scielo.mec.pt/scielo.php?script=sci_arttext\&pid=S08740492010000200003\&lng=pt\&tlng=pt.

Vrij, A., K. Edward, K. P. Roberts, \& R. Bull. (2000). Detecting deceit via analysis of verbal and nonverbal behavior. Journal of Nonverbal Behavior, 24, 239-263. doi: 10.1023/A:1006610329284.

Vrij, A., Akehurst, L., Soukara, S., \& Bull, R. (2004). Detecting deceit via analyses of verbal and nonverbal behavior in children and adults. Human Communication Research, 30, 8-41. doi: 10.1111/j.1468-2958.2004.tb00723.x. 
Vrij, A., Mann, S., \& Fisher, R. (2006). An empirical test of the behaviour analysis interview. Law and Human Behavior, 30, 329-345. doi: 10.1007/s10979-006-9014-3.

Vrij, A., Mann, S., Kristen, S., \& Fisher, R. (2007). Cues to deception and ability to detect lies as a function of police interview styles. Law and Human Behavior, 31(5), 499-518. doi: 10.1007/s10979-006-9066-4.

Vrij, A., Mann, S., Fisher, R., Leal, S., Milne, B., \& Bull, R. (2008). Increasing cognitive load to facilitate lie detection: The benefit of recalling an event in reverse order. Law and Human Behavior, 32, 253-265. doi: 10.1007/s10979-007-9103-y.

Vrij, A., Mann, S., Leal, S., \& Fisher, R. (2010). "Look into my eyes": Can an instruction to maintain eye contact facilitate lie detection? Psychology, Crime \& Law, 16, 327-348. doi: $10.1080 / 10683160902740633$.

Vrij, A., \& Ganis, G. (2014). Theories in Deception and Lie Detection. In D. C. Raskin, C. R. Honts, \& J. C. Kircher (Eds.), Credibility assessment (pp. 301-374). San Diego, CA: Academic Press (Elsevier).

Zuckerman, M., De Paulo, B. M., \& Rosenthal, R. (1981). Verbal and nonverbal communication of deception. In L. Berkowitz (Ed.), Advances in experimental social psychology (pp. 1-57). New York, EUA: Academic Press.

Zuckerman, M., Koestner, R., \& Alton, A. O. (1984). Learning to detect deception. Journal of Personality and Social Psychology, 46(3), 519-528. doi: 10.1037/00223514.46.3.519.

Submetido em: 03/12/2019

Aprovado em: 29/07/2020 